\begin{tabular}{|c|c|}
\hline Title & $\begin{array}{l}\text { Coexistence of High Electron Conduction and Low Heat Conduction in Tungsten Oxide Epitaxial Films with 1D } \\
\text { A tomic Defect Tunnels }\end{array}$ \\
\hline Author(s) & Kim, Gowoon; Feng, Bin; Sheu, Y u-Miin; Cho, Hai Jun; Ikuhara, Y uichi; Ohta, Hiromichi \\
\hline Citation & $\begin{array}{l}\text { A CS A pplied Electronic Materials, 2(8), 2507-2513 } \\
\text { https://doi.org/10.1021/acsaelm.0c00428 }\end{array}$ \\
\hline Issue Date & $2020-08-25$ \\
\hline Doc URL & http:/hdl.handle.net/2115/82516 \\
\hline Rights & $\begin{array}{l}\text { This document is the A ccepted Manuscript version of a Published Work that appeared in final form in ACS A pplied } \\
\text { Electronic Materials, copyright } \odot \text { A merican Chemical Society after peer review and technical editing by the publisher. } \\
\text { To access the final edited and published work see https://pubs.acs.org/doi } / 10.1021 / \text { acsaelm.0c00428. }\end{array}$ \\
\hline Tyре & article (author version) \\
\hline Additional Information & There are other files related to this item in HUSCAP. Check the above URL. \\
\hline File Information & Manuscript to A CS A ppl. Electron. Mater._revision_clean.pdf \\
\hline
\end{tabular}

Instructions for use 


\section{Coexistence of High Electron Conduction and Low Heat Conduction in Tungsten Oxide Epitaxial Films with 1D Atomic Defect Tunnels}

Gowoon Kim,* Bin Feng, Yu-Miin Sheu, Hai Jun Cho, Yuichi Ikuhara, and Hiromichi Ohta*

\section{Keywords}

thermal conductivity, electrical conductivity, tungsten oxide, 1D atomic defect tunnel, epitaxial film, pulsed laser deposition

ABSTRACT: Materials showing unusual electron and heat conduction such as the coexistence of high electron conduction and low heat conduction is essentially important to realize efficient thermal management systems. Although introducing point defects or layers is known as an effective way to reduce the thermal conductivity, the coexistence of high electron conduction and low heat conduction is still challenging because of the fact that electrons and phonons are scattered simultaneously by impurities, defects, and boundaries. Although oxygen-deficient tungsten oxide $\left(\mathrm{WO}_{x}\right)$ films $(2.7<x<3.0)$ show the desired properties, the origin is still unclear. Here we report that $1 \mathrm{D}$ atomic defect tunnels give rise to the coexistence of low thermal conductivity and high electrical conductivity of $\mathrm{WO}_{x}$ films. We fabricated $\mathrm{WO}_{x}$ epitaxial films on $\mathrm{LaAlO}_{3}$ substrates under a precisely controlled oxygen atmosphere. Crystallographic analyses revealed that $1 \mathrm{D}$ atomic defect tunnels are formed randomly along the rectangularshaped grains in the in-plane direction. The cross-plane thermal conductivity of the $\mathrm{WO}_{x}$ films dramatically decreased with decreasing $x$, while the electrical conductivity drastically increased due to an increase of carrier electrons, and high electron conduction and low heat conduction 
coexist when $x<2.9$. The present finding would be useful to design efficient thermal management materials.

\section{INTRODUCTION}

Electron conduction and heat conduction in media are associated with electrical conductivity, $\sigma$, and thermal conductivity, $\kappa$, respectively. $\sigma$ is a product of the carrier (electron) concentration and mobility. In the case of crystalline material, quantized lattice vibration (phonon) carries heat. In general, electron propagation is suppressed by phonons, impurities, atomic vacancies, and boundaries, and phonon propagation is suppressed by impurities, atomic vacancies, and boundaries. Since both $\sigma$ and $\kappa$ are increased/suppressed simultaneously by reducing/introducing impurities, atomic vacancies, and boundaries, it is considerably difficult to solely control the $\sigma$ or $\kappa$. Nevertheless, materials showing unusual electron and heat conduction such as the coexistence of high electron conduction and low heat conduction are required to realize efficient thermal management systems such as thermoelectrics. ${ }^{1-4}$

Nanostructuring approaches such as introducing point defects ${ }^{5-8}$ and/or layers ${ }^{9-11}$ are known as an effective way to reduce thermal conductivity. In general, a phonon mean free path is much longer than a carrier electron mean free path. Therefore, when the distance between two nearest neighbour defects is shorter than a phonon mean free path and longer than a carrier electron mean free path, phonon propagation would be suppressed but electron propagation would not be suppressed. ${ }^{2}$ However, it is still challenging to reduce heat conduction while keeping high electron conduction (mobility). The reason deters this approach from success can be understood by using the schematic diagram of Fig. 1. When a 0D defect or dot is introduced [Fig. 1(a)], both electron and phonon can be transported without suppression when the 0D defect density is low because of the low probability that the phonon hits the vacancies. On the other hand, both electrons and phonons are scattered when the $0 \mathrm{D}$ defect density is enough high ${ }^{12}$. If a 2D 
layered structure is fabricated [Fig. 1(c)], electrons and phonons are scattered at the heterointerfaces, therefore, they cannot be transported through the interface.

In order to overcome these difficulties, we propose an excellent solution [Fig. 1(b)]; introducing $1 \mathrm{D}$ atomic defect tunnel ${ }^{13-14}$ or wire to reduce the phonon propagation without reducing the electron propagation. In this structure, the probability of that phonon (mid-to-long wavelength ${ }^{2}$ ) hits the $1 \mathrm{D}$ atomic defect tunnel or wire is much higher than that of introducing $0 \mathrm{D}$ defect or dot even though the 1D defect density is low. Further, since the distance between two neighbouring $1 \mathrm{D}$ defects is much longer than the carrier electron mean free path, we expected that the electron propagation is not suppressed when the 1D defect density is low.

To verify this hypothesis experimentally, we selected oxygen-deficient tungsten oxide $\left(\mathrm{WO}_{x}\right.$, $2.7 \leq x \leq 3$ ) epitaxial films as the candidate. The bulk crystal structure of oxygen-deficient $\mathrm{WO}_{x}$ contains 1D tunnel structure ${ }^{15-16}$. $\mathrm{WO}_{x}$ can be epitaxially grown on perovskite oxide single crystals coherently. ${ }^{17-21}$ Therefore, we expected that $\mathrm{WO}_{x}$ with $1 \mathrm{D}$ tunnel structure can be stabilized on a substrate. In fact, Ning et al. ${ }^{21}$ reported that the thermal conductivity of $\mathrm{WO}_{x}$ films grown on (001) $\mathrm{LaAlO}_{3}$ substrate by modulating the oxygen atmosphere during the growth decreased dramatically with decreasing the $x$ whereas the electrical conductivity increased oppositely. They also showed that the thermal conductivity of $\mathrm{WO}_{x}$ decreased with increasing the electrical conductivity. Based on the results of the $\mathrm{WO}_{x}$ films grown on various substrates, they concluded that the thermal conductivity decreased with increasing the volumetric strain without discussing the atomic arrangement of the $\mathrm{WO}_{x}$ films. Although the thermal conductivity of a crystal is extremely sensitive to the disordering of the atomic arrangement, it has not been discussed yet. 
Here we show that $1 \mathrm{D}$ atomic defect tunnels originate the coexistence of low thermal conductivity and high electrical conductivity of $\mathrm{WO}_{x}$ films. We fabricated $\mathrm{WO}_{x}$ epitaxial films on $\mathrm{LaAlO}_{3}$ substrates under a precisely controlled oxygen atmosphere. Crystallographic analyses revealed that $1 \mathrm{D}$ atomic defect tunnels are formed randomly along the rectangularshaped grains in the in-plane direction. The cross-plane thermal conductivity of the $\mathrm{WO}_{x}$ films dramatically decreased with decreasing $x$, while the electrical conductivity drastically increased due to an increase of carrier electrons, and high electron conduction and low heat conduction coexist when $x<2.9$. The present finding would be useful to design efficient thermal management materials.

\section{RESULTS AND DISCUSSION}

The $\mathrm{WO}_{x}$ films were heteroepitaxially grown by the pulsed laser deposition (PLD) technique on (001) $\mathrm{LaAlO}_{3}$ single crystalline substrate at $700{ }^{\circ} \mathrm{C}$ under oxygen atmospheres. The oxygen pressure was varied from 2 to $13 \mathrm{~Pa}$ during the deposition to modulate the oxygen content $x$ in $\mathrm{WO}_{x}$. The $x$ in the $\mathrm{WO}_{x}$ films were successfully modulated from extracted from 2.787 to 2.977 as shown in Figs. 2(a) and 2(b), which were extracted from the X-ray photoelectron spectroscopy (XPS) spectra (Fig. S1). The volume fractions of $\mathrm{W}^{6+}$ and $\mathrm{W}^{5+}$ are dynamically changed with decreasing $x$ [Fig. 2(a)]. The composition $x$ in $\mathrm{WO}_{x}$ was determined from $\left(\left[\mathrm{W}^{6+}\right]\right.$ $\left.\times 3+\left[\mathrm{W}^{5+}\right] \times 2.5+\left[\mathrm{W}^{4+}\right] \times 2\right) /\left(\left[\mathrm{W}^{6+}\right]+\left[\mathrm{W}^{5+}\right]+\left[\mathrm{W}^{4+}\right]\right)^{22}$.

The film thickness was evaluated to be $\sim 40 \mathrm{~nm}$ in all cases from the analyses of the X-ray reflectivity. Coherent epitaxial growth was confirmed by measuring the X-ray reciprocal space mappings (Fig. S2); the in-plane lattice parameter is fixed with that of $\mathrm{LaAlO}_{3}$ substrate. Intense diffraction peak of WO with Pendellösung fringes is observed around $001 \mathrm{LaAlO}_{3}$ in the outof-plane XRD patterns [Fig. 2(c)], indicating a strong orientation of $\mathrm{WO}_{x}$. From the XRD results, lattice expansion from $\sim 0.366 \mathrm{~nm}$ to $\sim 0.385 \mathrm{~nm}$ was observed with decreasing $x$ [Fig. 2(d)]. 
The streak-like RHEED pattern [inset of Fig. 2(d)] was observed in the $\mathrm{WO}_{2.787}$ film, confirming that the film was heteroepitaxially grown. Checkerboard-like surface composed of rectangular-shaped grains ( $\sim 200 \mathrm{~nm}$ long, and $20 \mathrm{~nm}$ wide 1D wires, alternating in the plane to form checkboard-like pattern) was observed in the topographic AFM image of the $\mathrm{WO}_{2.787}$ film [Fig. 2(e)].

In order to visualize the atomic arrangement of the $\mathrm{WO}_{x}$ films directly, we performed the highangle annular dark-field (HAADF) scanning transmission electron microscopy (STEM) observations in the in-plane direction along the [100] $\mathrm{LaAlO}_{3}$ substrate (Fig. 3). Almost perfect square lattice is seen in the cross-sectional HAADF-STEM image of $\mathrm{WO}_{2.977}$ film [Fig. 3(a)], indicating that the film is epitaxially grown on (001) $\mathrm{LaAlO}_{3}$ substrate, which belongs to the epitaxially stabilized pseudo tetragonal structure. Misfit dislocation was not observed at the heterointerface between the film and the substrate, confirming the coherent epitaxial growth occurred. On the other hand, we found that the atomic defects were formed when $x$ is smaller than 2.955 in $\mathrm{WO}_{x}$ as shown in Figs. 3(b) and 3(f). The defects were randomly distributed. Since the images are the projection, these atomic defects are tunnel in shape. Further, Figs. 3(a)-3(e) show that the density of the atomic defect tunnels increased with decreasing of $x$ in $\mathrm{WO}_{x}$. In addition, the shape of the atomic defect tunnels became complex [Fig. 3(g)] when the density of the atomic defect tunnels increases, probably due to the interactions and relaxations between these defects that occurred during the film growth. It should be noted that the atomic arrangement of oxygen-deficient $\mathrm{WO}_{x}$ with many atomic defect tunnels looks random, like amorphous material. However, the atomic columns along with the electron beam direction are clearly visible, indicating the epitaxial growth continues even above the atomic defect tunnels [Figs. 3(f) and 3(g)]. The oxygen-deficient $\mathrm{WO}_{x}$ films receive stronger compressive strain from the substrate to forcibly stabilize the $\mathrm{WO}_{3}$ structure. Therefore, these atomic defect tunnels would be formed to release the strain during the film growth. 
It should be noted that the $1 \mathrm{D}$ atomic defect tunnels are located along the long axis of the rectangular-shaped grains; We observed the cross-sectional HAADF-STEM images from the direction along the long axis (data not shown). As a result, 1D atomic defect tunnels were clearly seen. On the other hand, from the direction across the long axis, a nearly perfect square lattice was seen (data not shown). Thus, the rectangular-shaped grains are single crystalline $\mathrm{WO}_{x}$ with $1 \mathrm{D}$ atomic defect tunnels. Although two different atomic arrangement is seen in the HAADF-STEM images due to that the thickness of the typical TEM sample is $\sim 100 \mathrm{~nm}$, it is reasonable because of the checkerboard-like arrangement of the rectangular-shaped grains $(L$ $\sim 200 \mathrm{~nm}, W \sim 20 \mathrm{~nm})$. These observations revealed that the 1D atomic defect tunnels are aligned along the long axis of the rectangular-shaped grains.

Here we demonstrate coexistence of high electron conduction and low heat conduction in $\mathrm{WO}_{x}$ epitaxial films with 1D atomic defect tunnels. The thermal conductivity $(\kappa)$ in the cross-plane direction drastically decreases with decreasing $x$ from 2.98 to 2.92 [Fig. 4(a)], whereas the electrical conductivity ( $\sigma_{\text {ave }}$ ) drastically increases simultaneously [Fig. 4(b)]. Very similar decreasing tendency is also seen in the $\kappa$ of $\mathrm{WO}_{x}$ epitaxial films grown on $\mathrm{LaAlO}_{3}$, which is reported by Ning et al. ${ }^{21}$ The $\kappa$ of $\mathrm{WO}_{2.97}$ was $\sim 7 \mathrm{~W} \mathrm{~m}{ }^{-1} \mathrm{~K}^{-1}$ and it decreased to $\sim 2 \mathrm{~W} \mathrm{~m}^{-1} \mathrm{~K}^{-1}$ with decreasing $x$ to 2.92, then gently decreased with decreasing $x$ (Figs. S3 and S4). The $\kappa$ values of the $\mathrm{WO}_{x}$ films $(x \leq 2.92)$ are close to that of amorphous $\mathrm{WO}_{x}$ film ${ }^{22}$, which shows minimum thermal conductivity ${ }^{23}$ among the same chemical composition. On the other hand, the measured $\sigma_{\text {ave }}$ in the in-plane direction of $\mathrm{WO}_{2.97}$ was $0.07 \mathrm{~S} \mathrm{~cm}^{-1}$ and it increased to $\sim 500$ $\mathrm{S} \mathrm{cm}^{-1}$ with decreasing $x$ to 2.92 . This is due to the increase of both carrier mobility and carrier electron concentration, which was extracted from thermopower analyses (Figs. S5 and S6). 
As schematically shown in Fig. 4(c), the $\kappa$ was measured in the cross-plane direction, whereas the $\sigma_{\text {ave }}$ was measured in the in-plane direction as shown in Fig. 4(d). Since the cross-plane electrical conductivity of the $\mathrm{WO}_{x}$ films cannot be directly measured, we measured the $1 \mathrm{D}$ electrical conductivities of several $\mathrm{WO}_{x}$ films with uniaxially oriented rectangular grains on (110) $\mathrm{LaAlO}_{3}$ substrates (Fig. S7). Many rectangular-shaped grains ( $L \sim 600 \mathrm{~nm}, W \sim 20 \mathrm{~nm}$ ) aligned in [001] direction are seen [Fig. S7(a)]. Although the azimuth of short axis of the rectangular grains is [1-10], which is different from that on (001) $\mathrm{LaAlO}_{3}$ substrates, the long axis is the same. Surprisingly, the electrical conductivities in the long axis of the rectangular grains, measured by d.c. four probe method as shown in Fig. S7(b), are almost the same as those on (001) $\mathrm{LaAlO}_{3}$ substrates [Fig. 4(b)]. Although the electrical conductivities in the short axis of the rectangular grains are a bit smaller than those in the long axis, the overall tendency of the electrical conductivity is similar to each other; the electrical conductivity increases with decreasing oxygen content. These results reveal that the coexistence of high electron conduction and low heat conduction in the oxygen-deficient $\mathrm{WO}_{x}$ crystals with the $1 \mathrm{D}$ atomic defect tunnel.

In addition, we analysed the low thermal conductivity of the $\mathrm{WO}_{x}$ epitaxial films by applying Wiedemann-Frantz (WF) law to extract phonon contribution and electron contribution to the heat propagation. In the case of crystalline metal, WF law can be applicable to extract the electron contribution to the observable thermal conductivity, $\kappa_{\mathrm{e}}$ as $\kappa_{e}=L \cdot \sigma \cdot T$, where $L$ is the Lorenz number, $\left(\pi^{2} / 3\right)\left(k_{\mathrm{B}} / e\right)^{2}=2.44 \times 10^{-8} \mathrm{~W} \Omega \mathrm{K}^{-2}$, and $T$ is the absolute temperature. Although WF law does not give a reliable $\kappa_{e}$ in the case of one of the transition metal oxides, $\mathrm{VO}_{2}{ }^{24}$, the $\kappa_{e}$ can be calculated in the case of the $\mathrm{WO}_{x}$ epitaxial films. We used the $\sigma_{\text {short }}$ values to extract the $\kappa_{e}$ in the cross-plane direction. The $\kappa_{e}$ and $\kappa_{l}$ of the $\mathrm{WO}_{2.92}$ film can be calculated as $\sim 0.07 \mathrm{~W} \mathrm{~m}^{-1} \mathrm{~K}^{-1}$ and $\sim 1.83 \mathrm{~W} \mathrm{~m}^{-1} \mathrm{~K}^{-1}$, close to that of amorphous $\mathrm{WO}_{2.93}\left(1.4 \mathrm{~W} \mathrm{~m}^{-1} \mathrm{~K}^{-1}\right)$, which is quite low thermal conductivity among low $\kappa$ oxides ${ }^{25}$. Although amorphous $\mathrm{WO}_{2.93}$ shows $\sim 10^{-1} \mathrm{~S} \mathrm{~cm}$ cm $^{-1}$, epitaxial $\mathrm{WO}_{2.92}$ shows three orders of magnitude large electrical 
conductivity. Thus, by introducing the 1D atomic defect tunnel, phonon propagation is suppressed but electron propagation is not suppressed. Since the 1D atomic defect tunnel is spontaneously introduced upon oxygen removal while keeping the epitaxial growth, and therefore, high electron conduction and low heat conduction are realized in the oxygen-deficient $\mathrm{WO}_{x}$ crystals.

\section{CONCLUSION}

In summary, we demonstrated that high electron conduction and low heat conduction are able to coexist simultaneously in an oxide crystal, oxygen-deficient $\mathrm{WO}_{x}$ epitaxial film. Crystallographic analyses revealed that the resultant $\mathrm{WO}_{x}$ films contain $1 \mathrm{D}$ atomic defect tunnels in the in-plane direction and the density increased with decreasing $x$. The 1D atomic defect tunnels were randomly distributed along the long axis of rectangular-shaped $\mathrm{WO}_{x}$ grains. The cross-plane thermal conductivity dramatically decreased with increasing the $1 \mathrm{D}$ atomic defect tunnels due to phonon propagation is suppressed, whereas the electrical conductivity dramatically increased simultaneously. High electron conduction and low heat conduction coexist when $x<2$ 2.92. The present finding would be useful to design efficient thermal management materials.

\section{EXPERIMENTAL PROCEDURES}

Fabrication and characterization of $\mathrm{WO}_{\mathrm{x}}$ epitaxial films with $1 \mathrm{D}$ atomic defect tunnel. The $\mathrm{WO}_{x}$ films were heteroepitaxially grown on (001) $\mathrm{LaAlO}_{3}$ single crystal substrate by pulsed laser deposition (PLD, KrF excimer laser, $\sim 1 \mathrm{~J} \mathrm{~cm}^{-2}$ pulse $^{-1}, 10 \mathrm{~Hz}$ ) at $700{ }^{\circ} \mathrm{C}$ in oxygen atmospheres. The oxygen pressure was varied from 2 to 13 Pa during the deposition to modulate the $x$ in $\mathrm{WO}_{x}$. The thickness of the films was $\sim 40 \mathrm{~nm}$ in all cases. High energy electron diffraction (RHEED) patterns were observed before exposing the film to the air. The growth condition of all the $\mathrm{WO}_{x}$ films was determined by optimizing the growth condition of the $\mathrm{WO}_{x}$ 
film (13 Pa) from the viewpoints of RHEED pattern, surface morphology, and average crystal tilting. Details of our PLD system have been described elsewhere. Valence states of W in the resultant $\mathrm{WO}_{x}$ films were analyzed by the X-ray photoelectron spectroscopy (XPS) using $\mathrm{Al}$ $\mathrm{K} \alpha$ radiation (1486.6eV). The flood gun was used to prevent charge up problem. The W $4 \mathrm{f}$ (30 $-42 \mathrm{eV}$ ) core level spectra were collected and all the spectra were calibrated with $\mathrm{C} 1 \mathrm{~s}$ peak at $284.8 \mathrm{eV}^{26}$. The thickness and the lattice parameters of the resultant films were analyzed by high-resolution X-ray diffraction ( $\mathrm{Cu} \mathrm{K} \alpha_{1}$, ATX-G, Rigaku Co.). X-ray reflectivity was measured to extract the density and thickness of the films. Out-of-plane Bragg diffraction patterns and X-ray reciprocal space mappings (RSMs) were recorded to measure the lattice parameters of the $\mathrm{WO}_{x}$ films. An atomic force microscopy (AFM, Nanocute, Hitachi Hi-Tech Sci. Co.) was used to observe the surface morphology of the films. The atomic arrangement of the films was visualized using high-angle annular dark-field scanning transmission electron microscopy (HAADF-STEM).

Electron transport properties. The electrical conductivity of the resultant $\mathrm{WO}_{x}$ films was measured by d.c. four-probe method. In-Ga alloy was used as the contact electrodes. Thermopower of the resultant films was measured by a standard steady-state method. The film sample was placed on the gap $(\sim 5 \mathrm{~mm})$ between two Peltier devices. By applying the forward/reverse current to each Peltier device, temperature differences were generated in the sample. We measured the temperature difference $(\Delta T)$ and the thermos-electromotive force $(\Delta V)$ simultaneously at room temperature. The thermopower was calculated as the linear slope of the $\Delta T-\Delta V$ plot.

Heat transport properties. Cross-plane thermal conductivity $(\kappa)$ of the resultant films was measured by the time domain thermo-reflectance (TDTR, PicoTR, PicoTherm Co.). The 116nm-thick Mo was deposited on the $\mathrm{WO}_{x}$ films as a transducer by dc sputtering. The decay curves 
of the TDTR signals were simulated using the packaged software developed by PicoTherm Co. In our simulation to minimize the number of free parameters, we used constant values that include bulk density $=10000 \mathrm{~kg} \mathrm{~m}^{-3}, C_{p}=250 \mathrm{~J} \mathrm{Kg}^{-1} \mathrm{~K}^{-1}$ and $\kappa=45.4 \mathrm{~W} \mathrm{~m}^{-1} \mathrm{~K}^{-1}$ for Mo

transducer and bulk density $=6520 \mathrm{~kg} \mathrm{~m}^{-3}, C_{p}=427 \mathrm{~J} \mathrm{Kg}^{-1} \mathrm{~K}^{-1}$ and $\kappa=10.3 \mathrm{~W} \mathrm{~m}^{-1} \mathrm{~K}^{-1}$ for the $\mathrm{LaAlO}_{3}$. These values were measured separately using a Mo transducer on the substrate. When the simulation includes a film, the film thickness and density were fixed by using the value derived from the XRR measurements. Because specific heat does not vary significantly for various $x$, we constrain the specific heat to allow small fluctuation around the reported single crystalline bulk values. ${ }^{27}$

\section{ASSOCIATED CONTENT}

\section{Supporting Information}

The Supporting Information is available free of charge via the Internet at http://pubs.acs.org.

XPS spectra around the $\mathrm{W} 4 \mathrm{f}$ of the $\mathrm{WO}_{x}$ epitaxial films; X-ray reciprocal space mappings of the $\mathrm{WO}_{x}$ epitaxial films; The time-dependent decay curves of the TDTR phase signal and fitted curve for the $\mathrm{WO}_{x}$ epitaxial films; In-plane electron transport properties of the $\mathrm{WO}_{x}$ epitaxial films; Calculated thermopower of the $\mathrm{WO}_{x}$ epitaxial films as a function of the carrier concentration; $1 \mathrm{D}$ electrical conductivity measurement of $\mathrm{WO}_{x}$ films with uniaxially oriented rectangular grains on (110) $\mathrm{LaAlO}_{3}$ substrates.

\section{AUTHOR INFORMATION}

\section{Corresponding Authors}

\section{Gowoon Kim}

Graduate School of Information Science and Technology, Hokkaido University, N14W9, Kita, Sapporo 060-0814, Japan 
ORCiD: orcid.org/0000-0002-5803-839X

rlarhdns32@eis.hokudai.ac.jp

\section{Hiromichi Ohta}

Research Institute for Electronic Science, Hokkaido University, N20W10, Kita, Sapporo 0010020, Japan

ORCiD: orcid.org/0000-0001-7013-0343

E-mail: hiromichi.ohta@es.hokudai.ac.jp

\section{Authors}

Bin Feng

Institute of Engineering Innovation, The University of Tokyo, 2-11-16 Yayoi, Bunkyo, Tokyo 113-8656, Japan

ORCiD: orcid.org/0000-0002-4306-2979

Yu-Miin Sheu

Center for Emergent Functional Matter Science, National Chiao Tung University, Hsinchu, 30010, Taiwan

ORCiD: orcid.org/0000-0002-4495-0708

Hai Jun Cho

Research Institute for Electronic Science, Hokkaido University, N20W10, Kita, Sapporo 0010020, Japan

ORCiD: orcid.org/0000-0002-8642-4183

Yuichi Ikuhara 
Institute of Engineering Innovation, The University of Tokyo, 2-11-16 Yayoi, Bunkyo, Tokyo 113-8656, Japan

ORCiD: orcid.org/0000-0003-3886-005X

\section{Author Contributions}

G.K. performed the sample preparation and measurements. B.F. and Y.I. performed the STEM analyses. G.K. and Y.S. analyzed the thermal conductivity. H.J.C. performed XPS analyses. G.K. and H.O. planned and supervised the project. All authors discussed the results and commented on the manuscript.

\section{Funding Sources}

Hiromichi Ohta received funding from Grants-in-Aid of JSPS (19H05791, 17H01314, and the Korea-Japan bilateral program), the Asahi Glass Foundation and the Mitsubishi Foundation. Bin Fen and Yuichi Ikuhara received funding from the MEXT Nanotechnology Platform (12024046). Gowoon Kim received funding from Grants-in-Aid for JSPS Fellows (2010147550) and the Asahi Glass Foundation Scholarship. Hai Jun Cho received funding from Nippon Sheet Glass Foundation for Materials Science and Engineering.

\section{Notes}

The authors declare no competing financial interests.

\section{ACKNOWLEDGEMENTS}

This research was supported by Grants-in-Aid for Innovative Areas (19H05791 and 19H05788) from the JSPS. A part of this work was supported by Dynamic Alliance for Open Innovation Bridging Human, Environment, and Materials, and by the Network Joint Research Center for Materials and Devices. A part of this work was also supported by the "Nanotechnology 
Platform” (12024046) of the MEXT (B.F. and Y.I.). G.K. was supported by Grants-in-Aid for JSPS Fellows (2010147550) from JSPS and the Asahi Glass Foundation Scholarship. H.J.C. acknowledges the support from Nippon Sheet Glass Foundation for Materials Science and Engineering. H.O. was supported by Grants-in-Aid for Scientific Research A (17H01314) from the JSPS, the Asahi Glass Foundation and the Mitsubishi Foundation.

\section{REFERENCES}

(1) Snyder, G. J.; Toberer, E. S., Complex thermoelectric materials. Nature Mater. 2008, 7

(2), 105-114.

(2) Vineis, C. J.; Shakouri, A.; Majumdar, A.; Kanatzidis, M. G., Nanostructured

Thermoelectrics: Big Efficiency Gains from Small Features. Advanced Materials 2010, 22 (36), 3970-3980.

(3) He, J.; Tritt, T. M., Advances in thermoelectric materials research: Looking back and moving forward. Science 2017, 357 (6358), eaak9997.

(4) Poudel, B.; Hao, Q.; Ma, Y.; Lan, Y. C.; Minnich, A.; Yu, B.; Yan, X. A.; Wang, D. Z.; Muto, A.; Vashaee, D.; Chen, X. Y.; Liu, J. M.; Dresselhaus, M. S.; Chen, G.; Ren, Z. F., High-thermoelectric performance of nanostructured bismuth antimony telluride bulk alloys. Science 2008, 320 (5876), 634-638.

(5) Hsu, K. F.; Loo, S.; Guo, F.; Chen, W.; Dyck, J. S.; Uher, C.; Hogan, T.; Polychroniadis, E.; Kanatzidis, M. G., Cubic $\mathrm{AgPb}_{m} \mathrm{SbTe}_{2+m}$ : bulk thermoelectric materials with high figure of merit. Science 2004, 303 (5659), 818-821.

(6) Luckyanova, M. N.; Chen, D.; Ma, W.; Tuller, H. L.; Chen, G.; Yildiz, B., Thermal conductivity control by oxygen defect concentration modification in reducible oxides: The case of $\operatorname{Pr}_{0.1} \mathrm{Ce}_{0.9} \mathrm{O}_{2-\delta}$ thin films. Appl. Phys. Lett. 2014, 104 (6), 061911.

(7) Scott, E. A.; Hattar, K.; Rost, C. M.; Gaskins, J. T.; Fazli, M.; Ganski, C.; Li, C.; Bai, T.; Wang, Y.; Esfarjani, K.; Goorsky, M.; Hopkins, P. E., Phonon scattering effects from point 
and extended defects on thermal conductivity studied via ion irradiation of crystals with selfimpurities. Phys. Rev. Mater. 2018, 2 (9), 095001.

(8) Dongre, B.; Carrete, J.; Wen, S.; Ma, J.; Li, W.; Mingo, N.; Madsen, G. K. H., Combined treatment of phonon scattering by electrons and point defects explains the thermal conductivity reduction in highly-doped Si. J. Mater. Chem. A 2020, 8, 1273-1278.

(9) Venkatasubramanian, R.; Siivola, E.; Colpitts, T.; O'Quinn, B., Thin-film thermoelectric devices with high room-temperature figures of merit. Nature 2001, 413 (6856), 597-602. (10) Ravichandran, J.; Yadav, A. K.; Cheaito, R.; Rossen, P. B.; Soukiassian, A.; Suresha, S.; Duda, J. C.; Foley, B. M.; Lee, C. H.; Zhu, Y.; Lichtenberger, A. W.; Moore, J. E.; Muller, D. A.; Schlom, D. G.; Hopkins, P. E.; Majumdar, A.; Ramesh, R.; Zurbuchen, M. A., Crossover from incoherent to coherent phonon scattering in epitaxial oxide superlattices. Nat. Mater. 2014, 13 (2), 168-172.

(11) Dechaumphai, E.; Lu, D.; Kan, J. J.; Moon, J.; Fullerton, E. E.; Liu, Z.; Chen, R., Ultralow thermal conductivity of multilayers with highly dissimilar debye temperatures. Nano Lett. 2014, 14 (5), 2448-2455.

(12) Sy, H.; Ong, C., Electron mobility in heavily doped silicon. Solid State Commun. 1984, 52 (10), 881-883.

(13) Yuan, Y.; Wood, S. M.; He, K.; Yao, W.; Tompsett, D.; Lu, J.; Nie, A.; Islam, M. S.; Shahbazian-Yassar, R., Atomistic insights into the oriented attachment of tunnel-based oxide nanostructures. ACS nano 2016, 10 (1), 539-548.

(14) Yin, D.; Chen, C.; Saito, M.; Inoue, K.; Ikuhara, Y., Ceramic phases with onedimensional long-range order. Nat. Mater. 2019, 18 (1), 19-23.

(15) Magnéli, A., Tungsten Bronzes containing Six-membered Rings of $\mathrm{WO}_{6}$ Octahedra. Nature 1952, 169 (4306), 791-792.

(16) Mardare, C. C.; Hassel, A. W., Review on the Versatility of Tungsten Oxide Coatings. Phys. Status Solidi A 2019, 216 (12), 1900047. 
(17) Leng, X.; Pereiro, J.; Strle, J.; Bollinger, A.; Božović, I., Epitaxial growth of high quality $\mathrm{WO}_{3}$ thin films. APL Mater. 2015, 3 (9), 096102.

(18) Nishihaya, S.; Uchida, M.; Kozuka, Y.; Iwasa, Y.; Kawasaki, M., Evolution of InsulatorMetal Phase Transitions in Epitaxial Tungsten Oxide Films during Electrolyte-Gating. ACS Appl. Mater. Interfaces 2016, 8 (34), 22330.

(19) Wang, M.; Shen, S.; Ni, J.; Lu, N.; Li, Z.; Li, H. B.; Yang, S.; Chen, T.; Guo, J.; Wang, Y.; Xiang, H.; Yu, P., Electric - Field - Controlled Phase Transformation in $\mathrm{WO}_{3}$ Thin Films through Hydrogen Evolution. Adv. Mater. 2017, 29 (46), 1703628.

(20) Mattoni, G.; Filippetti, A.; Manca, N.; Zubko, P.; Caviglia, A. D., Charge doping and large lattice expansion in oxygen-deficient heteroepitaxial $\mathrm{WO}_{3}$. Phys. Rev. Mater. 2018, 2 (5), 053402.

(21) Ning, S.; Huberman, S. C.; Ding, Z.; Nahm, H. H.; Kim, Y. H.; Kim, H. S.; Chen, G.; Ross, C. A., Anomalous Defect Dependence of Thermal Conductivity in Epitaxial $\mathrm{WO}_{3}$ Thin Films. Advanced Materials 2019, 31 (43), 1903738.

(22) Kim, G.; Cho, H. J.; Sheu, Y.-M.; Ohta, H., Electrical, Optical and Thermal Transport Properties of Oxygen Deficient Amorphous $\mathrm{WO}_{x}(2.5<x<3)$ Films. J. Phys. Chem. C 2019, $123,15419-15424$.

(23) Agne, M. T.; Hanus, R.; Snyder, G. J., Minimum thermal conductivity in the context of diffuson-mediated thermal transport. Energy Environ. Sci. 2018, 11 (3), 609-616.

(24) Lee, S.; Hippalgaonkar, K.; Yang, F.; Hong, J.; Ko, C.; Suh, J.; Liu, K.; Wang, K.; Urban, J. J.; Zhang, X.; Chris, D.; Hartnoll, S. A.; Delaire, O.; Wu, J., Anomalously low electronic thermal conductivity in metallic vanadium dioxide. Science 2017, 355 (6323), 371374.

(25) Winter, M. R.; Clarke, D. R., Oxide materials with low thermal conductivity. J. Am. Ceram. Soc. 2007, 90 (2), 533-540. 
(26) Swift, P., Adventitious carbon—-the panacea for energy referencing? Surf. Interface Anal. 1982, 4 (2), 47-51.

(27) Sale, F., Heat capacities of the tungsten oxides $\mathrm{WO}_{3}, \mathrm{~W}_{20} \mathrm{O}_{58}, \mathrm{~W}_{18} \mathrm{O}_{49}$ and $\mathrm{WO}_{2}$.

Thermochim. Acta 1979, 30 (1-2), 163-171. 


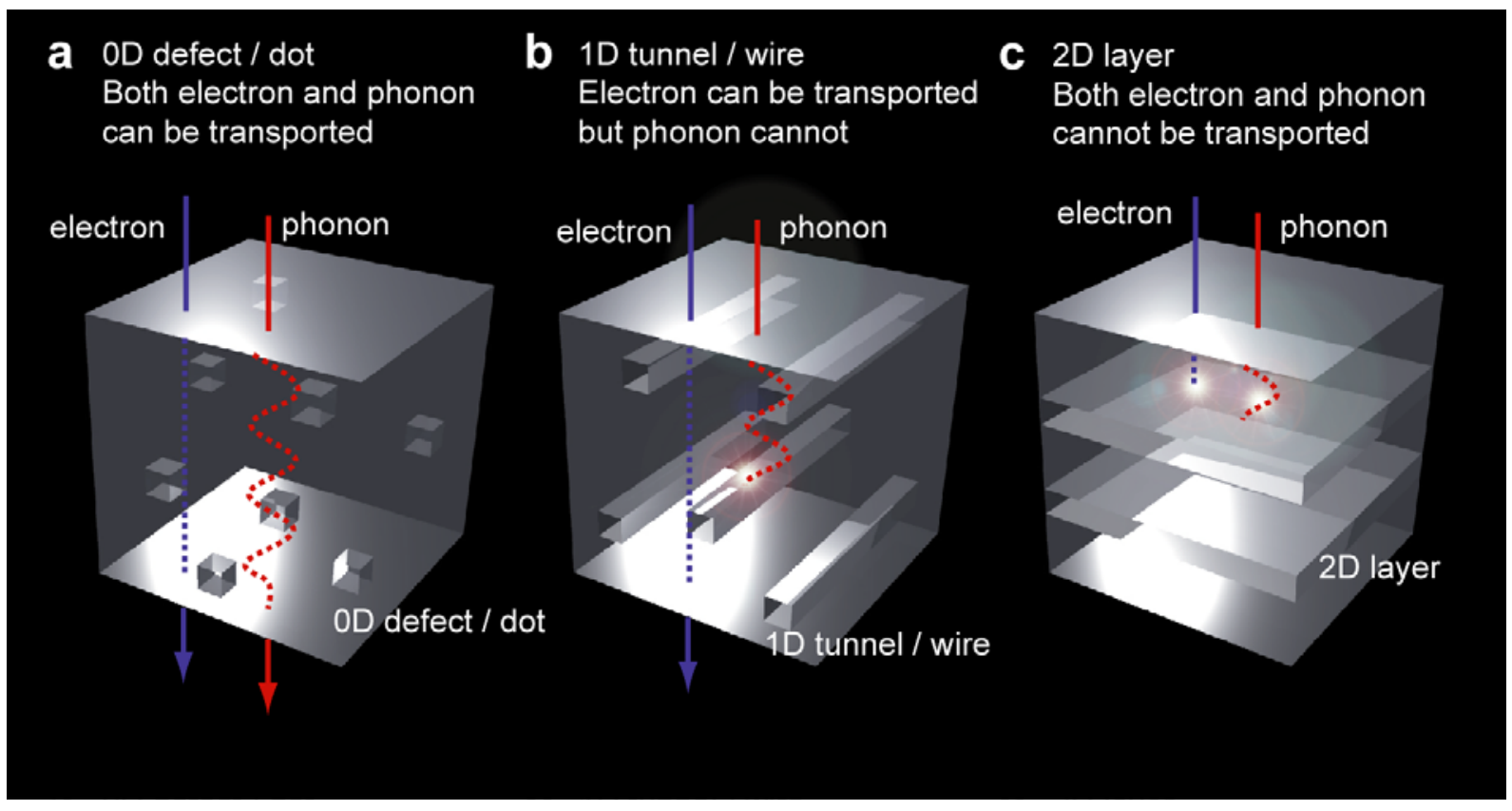

Figure 1. Nanostructuring concept to reduce heat conduction while keeping high electron conduction. Schematic illustrations of electron/phonon propagation in materials with (a) 0D defect (dot), (b) 1D tunnel (wire), and (c) 2D layer. (a) Electron and phonon can be transported without suppression because of the low probability of that phonon hits the vacancies. Electron transport is less sensitive to the defect. (b) the probability of that phonon hits the 1D tunnel is much higher than that in 0D. Therefore, low thermal conductivity can be expected. (c) 2D layer insertion is effective to reduce thermal conductivity. However, electrons are also scattered at the interface, which results in low electrical conductivity and low thermal conductivity. 

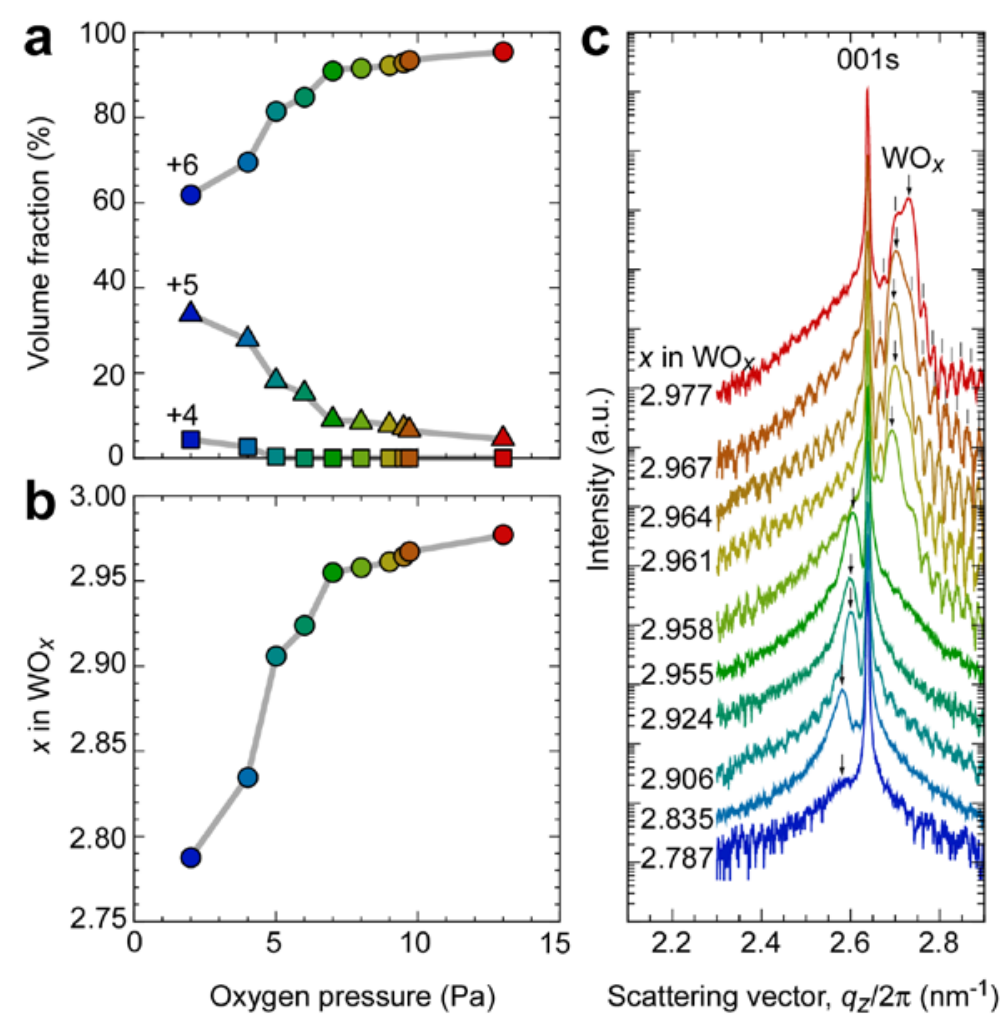
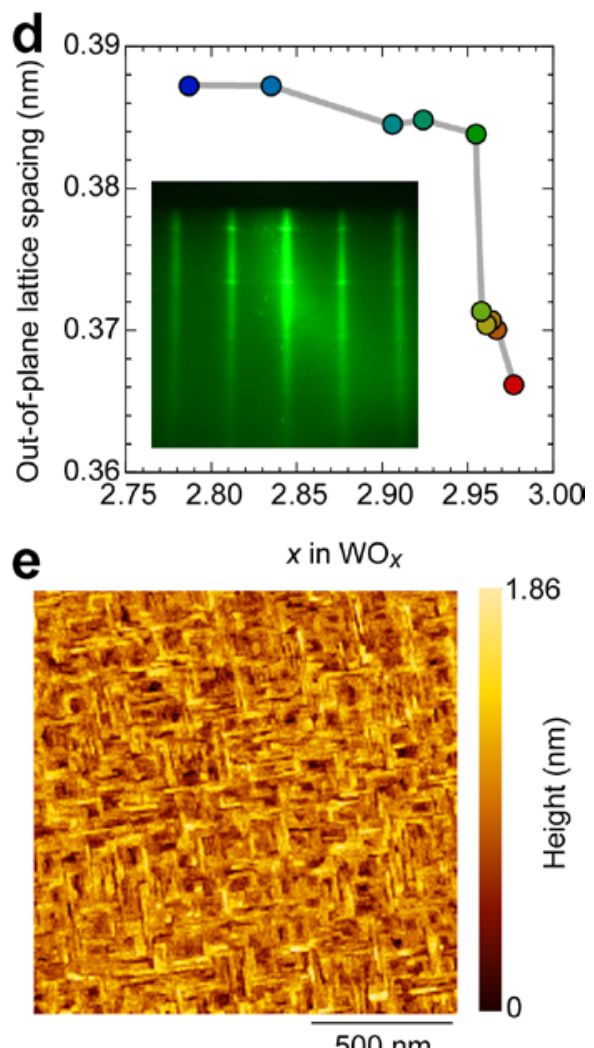

$500 \mathrm{~nm}$

Figure 2. Change in the crystalline lattice of the $\mathrm{WO}_{x}$ epitaxial films. (a) Volume fraction of $\mathrm{W}^{6+}, \mathrm{W}^{5+}$, and $\mathrm{W}^{4+}$ and (b) $x$ in the $\mathrm{WO}_{x}$ films as a function of the oxygen pressure during the film growth. The $x$ in $\mathrm{WO}_{x}$ was modulated from $\sim 2.78$ to $\sim 2.98$. (c) Out-of-plane XRD patterns of the $\mathrm{WO}_{x}$ films around $001 \mathrm{LaAlO}_{3}$. The arrows $(\downarrow)$ indicate the diffraction peak position of $\mathrm{WO}_{x}$ and the short lines ( $\mid$ ) indicate Pendellösung fringes. (d) Change in the out-of-plane lattice spacing of $\mathrm{WO}_{x}$. The inset shows the RHEED pattern of the $\mathrm{WO}_{2.787}$ film, confirming the successful heteroepitaxial growth. (e) Topographic AFM image of the $\mathrm{WO}_{2.787}$ film. Checkerboard-like arrangement of the rectangular-shaped grains $(L \sim 200 \mathrm{~nm}, W \sim 20 \mathrm{~nm})$ is seen. 
a

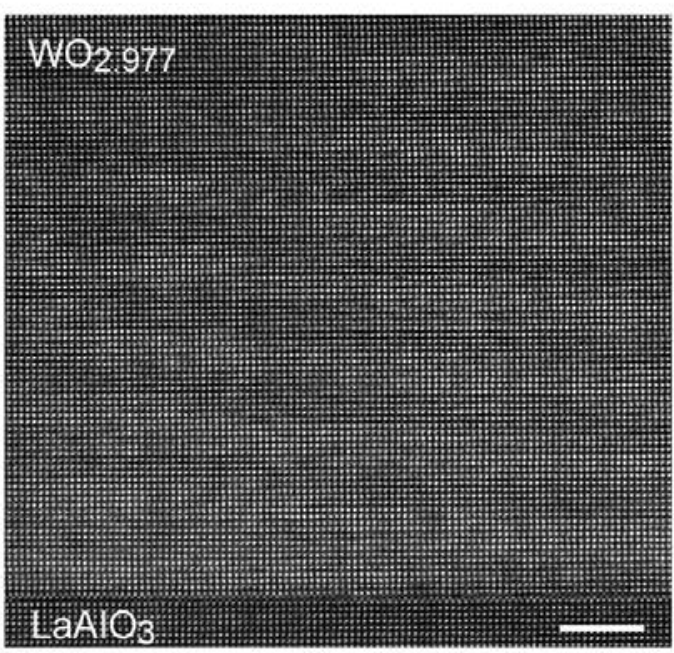

C

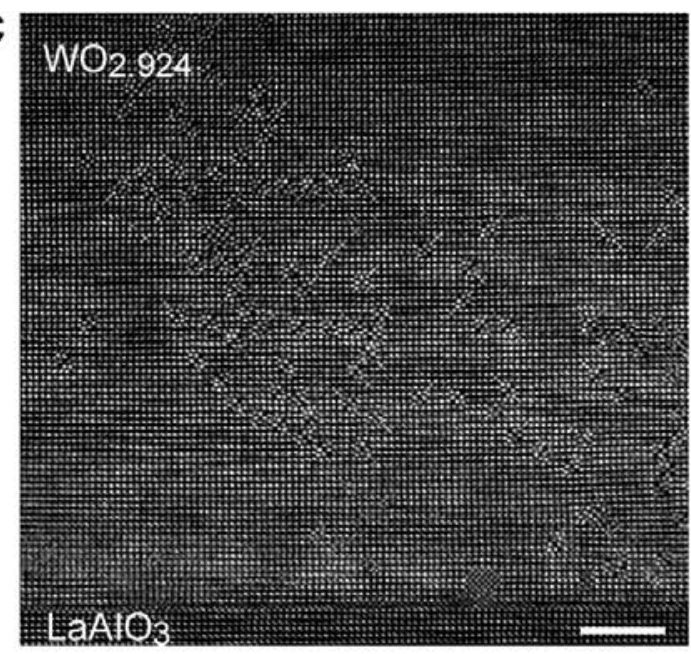

e

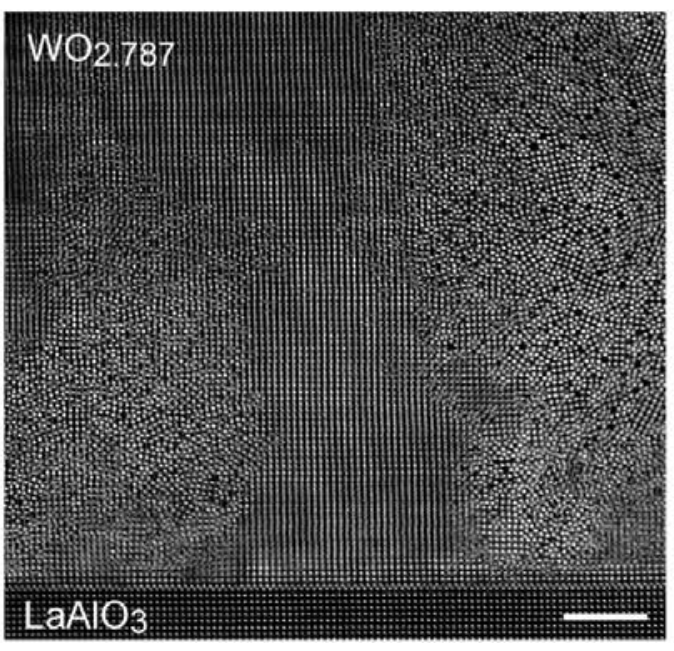

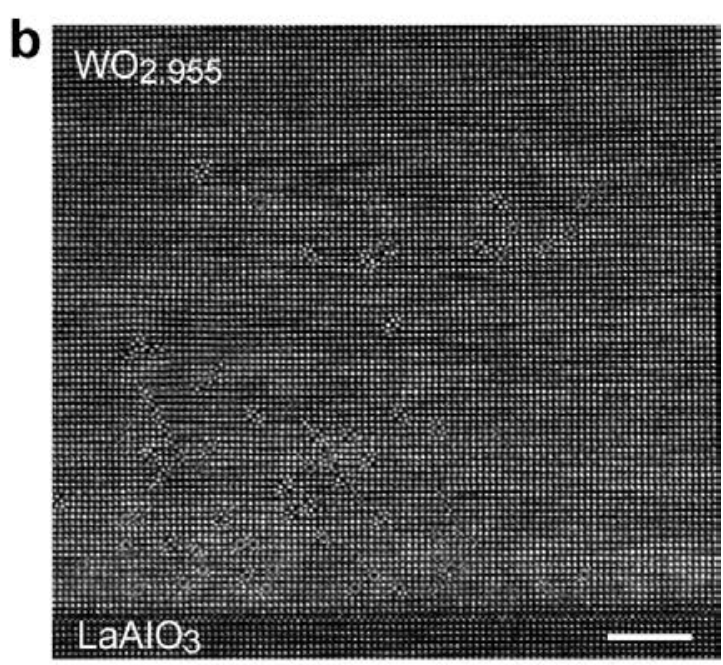
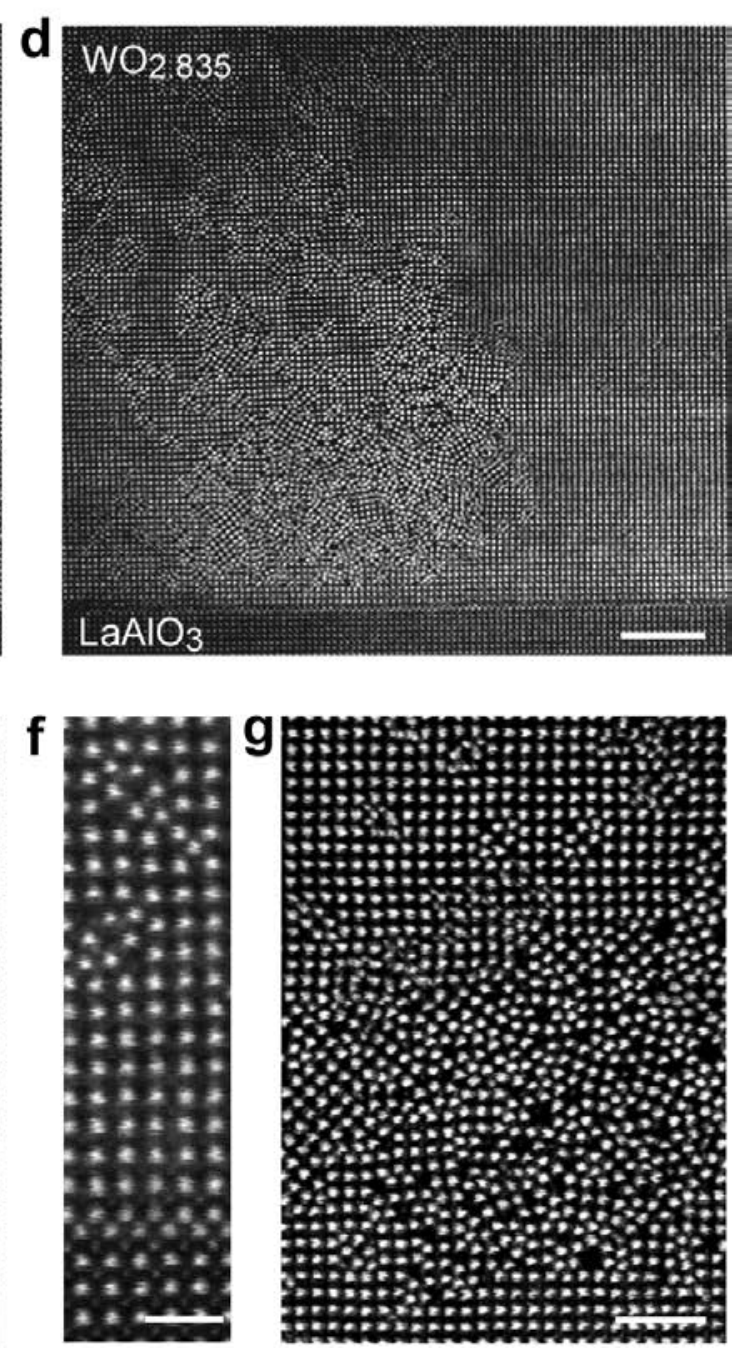

Figure 3. Evolution of $1 \mathrm{D}$ atomic defect tunnel in the oxygen-deficient (001) $\mathrm{WO}_{x}$ epitaxial films. Cross-sectional HAADF-STEM images of (a) $\mathrm{WO}_{2.977}$, (b) $\mathrm{WO}_{2.955}$, (c) $\mathrm{WO}_{2.924}$, (d) $\mathrm{WO}_{2.835}$, and (e) $\mathrm{WO}_{2.787}$ films. (f) Atomic structure of the $1 \mathrm{D}$ atomic defects in (b). (g) Atomic structure of high-density 1D atomic defect in (e). The scale bar is $5 \mathrm{~nm}$ for (a)-(e), $1 \mathrm{~nm}$ for (f), $2 \mathrm{~nm}$ for (g). 


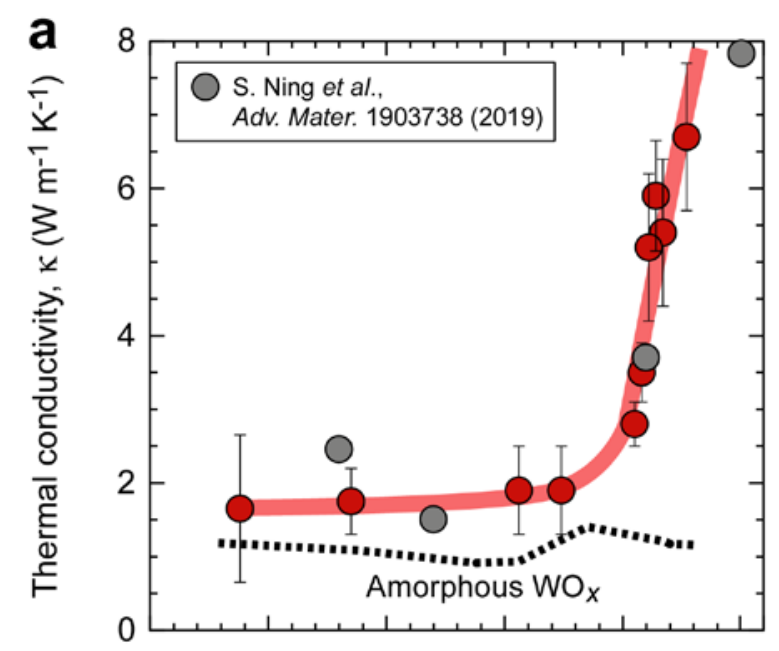

C Cross-plane thermal conductivity
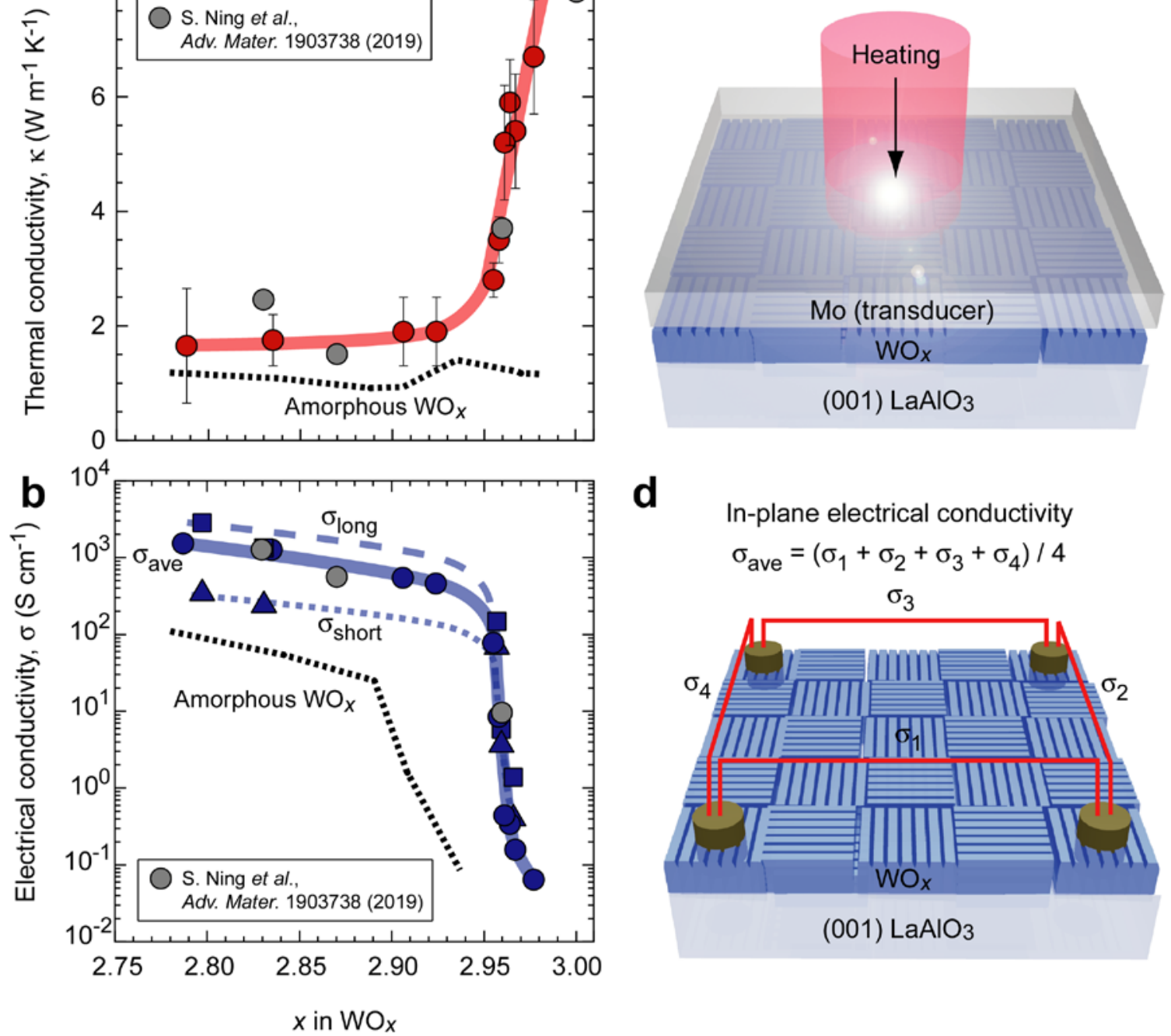

d

In-plane electrical conductivity

$\sigma_{\text {ave }}=\left(\sigma_{1}+\sigma_{2}+\sigma_{3}+\sigma_{4}\right) / 4$

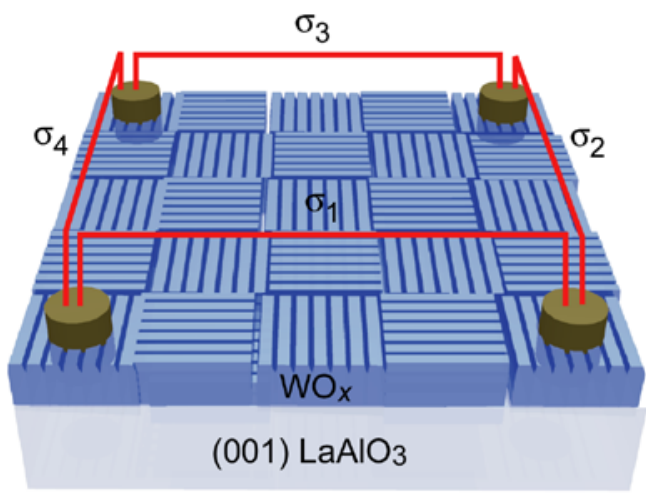

Figure 4. Coexistence of high electron conduction and low heat conduction in the $\mathrm{WO}_{x}$ epitaxial films with 1D atomic defect tunnels. (a) Cross-plane thermal conductivity $\kappa$ extracted from the TDTR measurement. The $\kappa$ of amorphous $\mathrm{WO}_{x}$ films ${ }^{22}$ and epitaxial $\mathrm{WO}_{x}$ films ${ }^{21}$ are also plotted. (b) In-plane electrical conductivity $\sigma$. The $\sigma$ of amorphous $\mathrm{WO}_{x}$ films $^{22}$ and epitaxial $\mathrm{WO}_{x}$ films ${ }^{21}$ are also plotted. (c) Schematic illustration of the TDTR measurement of the $\mathrm{WO}_{x}$ films. (d) Schematic illustration of the electrical conductivity measurement of the $\mathrm{WO}_{x}$ films. 